\title{
Subacute Cutaneous Lupus Erythematosus Triggered by Radiotherapy
}

\author{
I. Kolm E. Pawlik N. Eggmann J. Kamarachev K. Kerl L.E. French \\ G.F.L. Hofbauer \\ Department of Dermatology, University Hospital Zurich, Zurich, Switzerland
}

\section{Key Words}

Lupus erythematosus $\cdot$ Radiotherapy $\cdot$ Autoimmunity $\cdot$ Autoimmune reaction

\begin{abstract}
Background: The origin of collagen autoimmune diseases is not fully understood. Some studies postulate a mechanism of molecular mimicry or heterologous immunity following viral infections triggering autoimmunity. Apart from infections, other exogenous factors such as visible light or X-rays have been reported to incite autoimmunity. Case Report: We report a case of histologically and serologically confirmed subacute lupus erythematosus (SCLE) following radiotherapy for breast cancer. Discussion: The close temporal and spatial correlation between radiotherapy and onset of SCLE in this patient suggests that an autoimmune reaction may have been triggered locally by functionally altering the immune system and breaking self-tolerance.

(c) 2013 S. Karger AG, Basel
\end{abstract}

\section{Case Report}

A 72-year-old woman underwent breast cancer surgery and received adjuvant systemic treatment with the estrogen receptor antagonist tamoxifen $20 \mathrm{mg} /$ day, which was discontinued after the patient developed a generalized skin rash about two weeks after the initiation of tamoxifen. This rash was clinically and histologically diagnosed as erythema exsudativum multiforme, which rapidly resolved under systemic treatment with prednisolone. Tamoxifen was discontinued. The patient afterwards received radiotherapy of the left breast. A cumulative dose of 40 Gy in 15 fractions was administered and was initially well

Isabel Kolm, MD

Department of Dermatology, University Hospital Zurich

Gloriastrasse 31

$\mathrm{CH}-8091$ Zürich (Switzerland)

E-Mail Isabel.Kolm@usz.ch 
tolerated by the patient. After finishing radiotherapy, our patient developed a painless maculopapular erythema on the left breast limited to the irradiated area.

Histology of a skin biopsy taken from the left breast showed an acute interface dermatitis with vacuolization of the dermo-epidermal junction, apoptotic keratinocytes in the basal epidermis and a mild, predominantly lymphocytic, infiltrate within the superficial dermis and with few admixed neutrophils and eosinophilic granulocytes.

These histological changes were consistent with subacute cutaneous lupus erythematosus (SCLE). Because of the presence of eosinophilic granulocytes in the inflammatory infiltrate, a fixed drug eruption was considered a differential diagnosis. An allergic reaction to tamoxifen was ruled out by a negative scratch-patch test.

Serology revealed elevated titers for antinuclear antibodies 1:2,560, anti-SS-A at $>3,400$ $\mathrm{U} / \mathrm{ml}$, anti-SS-B at $>3,400 \mathrm{U} / \mathrm{ml}$ and rheumatoid factor at $41 \mathrm{IU} / \mathrm{ml}$. Complement components (C3 and C4) were within normal limits and urine analysis was unremarkable.

Based on the patient's history, the clinical presentation of the skin lesions, serologic results, histopathology and allergy testing, a diagnosis of SCLE - presumably triggered by radiotherapy - was made.

The administration of topical corticosteroids and systemic hydroxychloroquine at 400 $\mathrm{mg} /$ day led to a total disappearance of the lesions on the left breast within 6 weeks.

\section{Discussion}

SCLE was first characterized by Sontheimer et al. [1] in 1979 as a recurring, nonfixed, superficial, nonscarring, photosensitive form of lupus erythematosus with a papulosquamous or an anular pattern, lupus-specific histopathology, lack of systemic involvement and a high association with circulating antinuclear and anti-SS-A in $80 \%$ antibodies, the latter occurring of the cases. Anti-SS-B auto-antibodies are found less frequently. Direct immunofluorescence remains negative in up to $40 \%$ of the cases [1, 2].

Triggering agents for SCLE have been well recognized. Best described is the impact of UV light on the development of SCLE [3]. Regarding our patient, this important trigger could be ruled out.

Apart from phototriggering, many drugs have been related to the induction of SCLE. They include NSAID, tetracyclines and antihypertensive drugs among many others. Fumal et al. [4] previously described two cases of tamoxifen-induced SCLE starting after several years of treatment with tamoxifen. Tamoxifen seems to be an unlikely trigger factor for SCLE in our patient because tamoxifen had been discontinued two months before the appearance of the skin lesions on the breast. Additionally, scratch-patch testing for tamoxifen was negative.

Another differential diagnosis includes a rare and controversial entity called Rowell's syndrome, which describes the clinical association of lupus erythematosus with erythema multiforme-like lesions and a speckled pattern of antinuclear antibodies (major criteria). The minor criteria include chilblains, anti-Ro and/or anti-La antibodies and positive rheumatoid factor. All three major criteria and at least one minor criteria are required to establish the diagnosis of Rowell's syndrome. The clinical and serological criteria (histological findings consistent with erythema multiforme and SCLE, elevated titers for antinuclear antibodies $1: 2,560$, anti-SS-A at $>3,400 \mathrm{U} / \mathrm{ml}$, anti-SS-B at $>3,400 \mathrm{U} / \mathrm{ml}$ and rheumatoid factor at $41 \mathrm{IU} / \mathrm{ml}$ ) provide strong evidence for the diagnosis of Rowell's syndrome in our case but would not explain the limitation of SCLE to the irradiation site on the left breast two months after the appearance of the erythema multiforme. 
Radiotherapy most frequently induces acute radiation dermatitis. Other acute cutaneous reactions to radiotherapy are contact dermatitis, Sweet's syndrome, lichen planus, pemphigus, cutaneous hypersensitivity syndromes such as erythema multiforme as well as nonspecific hypersensitivity reactions like urticaria, localized Stevens-Johnson syndrome or even toxic epidermal necrolysis.

Radiation recall dermatitis refers to the rare appearance of a maculopapular rash in previously irradiated skin after the administration of drugs, especially chemotherapeutic agents or antibiotics $[5,6]$. Radiation recall dermatitis typically develops within days or weeks after the administration of the drug. The diagnosis of radiation recall dermatitis in our case can be ruled out as tamoxifen was stopped prior to radiotherapy.

Fleck et al. [7] reported the association of radiotherapy with collagen vascular disorders such as CREST, discoid lupus, acute lupus erythematosus, Sjögren's syndrome and dermatomyositis in patients with preexisting collagen diseases. So far, radiotherapy inducing SCLE has been described in the literature only once before: Balabanova et al. [8] reported on an elderly female patient suffering from nondifferentiated lobular breast carcinoma with metastases in the axillary lymph nodes who developed an erythematous scaly eruption of the left thoracic area following radiotherapy of the area and was clinically, immunologically and histologically diagnosed as having SCLE.

\section{Conclusion}

The ethiopathogenesis of SCLE skin lesions is the result of 4 sequential stages: (1) inheritance of susceptibility genes; (2) loss of tolerance/induction of autoimmunity; (3) expansion/maturation of autoimmune responses, and (4) tissue injury/disease induction resulting from various autoimmune effector mechanisms [9]. It has been described that ionizing radiation can functionally alter the immune system and break self-tolerance [10]. In our case, the close temporal and spatial correlation between radiotherapy and onset of SCLE suggests that an autoimmune reaction may have been triggered locally, e.g. by the presentation of an auto-antigen and the induction of a danger milieu favoring immune recognition in the setting of radiodermatitis [11]. While we lack formal proof for this interpretation, the patient's history and further workup exclude most other reported associations, leaving us with radiotherapy as the most likely trigger of SCLE in this case.

\section{Disclosure Statement}

The authors declare no conflict of interest.

\section{References}

1 Sontheimer RD, Thomas JR, Gilliam JN: Subacute cutaneous lupus erythematosus: a cutaneous marker for a distinct lupus erythematosus subset. Arch Dermatol 1979;115:1409-1415.

-2 Sontheimer RD, Maddison PJ, Reichlin M, Jordon RE, Stastny P, Gilliam JN: Serologic and HLA associations in subacute cutaneous lupus erythematosus, a clinical subset of lupus erythematosus. Ann Intern Med 1982;97:664-671.

-3 Kuhn A, Sonntag M, Richter-Hintz D, Oslislo C, Megahed M, Ruzicka T, Lehmann P: Phototesting in lupus erythematosus: a 15-year experience. J Am Acad Dermatol 2001;45:86-95.

-4 Fumal I, Danchin A, Cosserat F, Barbaud A, Schmutz JL: Subacute cutaneous lupus erythematosus associated with tamoxifen therapy: two cases. Dermatology 2005;210:251-252. 
Kolm et al.: Subacute Cutaneous Lupus Erythematosus Triggered by Radiotherapy

5 Hymes SR, Strom EA, Fife C: Radiation dermatitis: clinical presentation, pathophysiology, and treatment 2006. J Am Acad Dermatol 2006;54:28-46.

6 Baek SW, Seo YJ, Kim JS, Lee HJ: Radiation recall dermatitis after treatment with Paclitaxel and Cisplatin. Ann Dermatol 2012;24:223-224.

7 Fleck R, McNeese MD, Ellerbroeck NA, Hunter TA, Holmes FA: Consequences of breast irradiation in patients with pre-existing collagen vascular disease. Int J Radiat Oncol Biol Phys 1989;17:829-833.

8 Balabanova MB, Botev IN, Michailova JI: Subacute cutaneous lupus erythematosus induced by radiation therapy. Br J Dermatol 1997;137:648-649.

-9 Sontheimer R: Subacute cutaneous lupus erythematosus: 25-year evolution of a prototypic subset (subphenotype) of lupus erythematosus defined by characteristic cutaneous, pathological, immunological and genetic findings. Autoimmun Rev 2005;4:253-263.

10 Sakaguchi N, Miyai K, Sakaguchi S: Ionizing radiation and autoimmunity. Induction of autoimmune disease in mice by high dose fractionated total lymphoid irradiation and its prevention by inoculating normal $\mathrm{T}$ cells. J Immunol 1994;152:2586-2595.

11 Matzinger P: An innate sense of danger. Ann NY Acad Sci 2002;961:341-342.

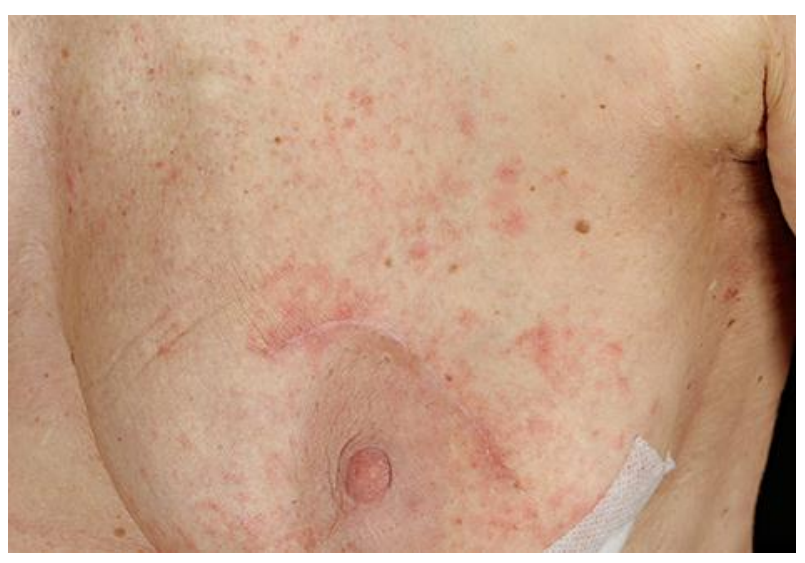

Fig. 1. Maculopapular eruption appearing after radiotherapy limited to the irradiation site on the left breast. 


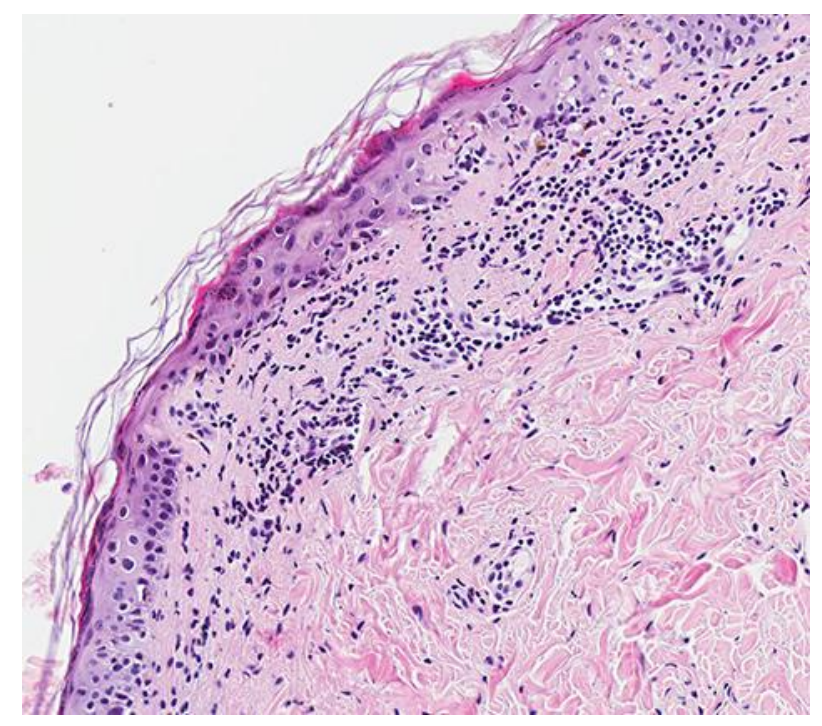

Fig. 2. Histopathology of a papule within the affected area of the left breast showing acute interface dermatitis with vacuolization of the dermo-epidermal junction, apoptotic keratinocytes in the basal epidermis and a mild predominantly lymphocytic infiltrate within the superficial dermis with a few admixed neutrophils and eosinophilic granulocytes. 